\title{
Statistical approximation for multivariable integrable functions
}

\author{
İlknur Sakaoğlu and Mehmet Ünver
}




\title{
STATISTICAL APPROXIMATION FOR MULTIVARIABLE INTEGRABLE FUNCTIONS
}

\author{
İLKNUR SAKAOĞLU AND MEHMET ÜNVER
}

Received 16 February, 2011

\begin{abstract}
Using the concept of $A$-statistical convergence we give Korovkin type approximation theorems for a sequence of $A$-statistically uniformly bounded positive linear operators acting from $L_{p}[a, b ; c, d]$ into itself.
\end{abstract}

2000 Mathematics Subject Classification: 41A36; 47B38

Keywords: $A$-statistical convergence, Korovkin type theorem, Multivariable integrable function

\section{INTRODUCTION}

Recall that Korovkin type approximation theory deals with the problem of approximating a function $f$ by a sequence $\left\{T_{n}(f, x)\right\}$ of positive linear operators over a certain space of real valued functions (see, e.g, [1], [10]). In particular, this type of results in the space $L_{p}[a, b]$ of integrable functions on a compact interval may be found in [2], [3], [6], [11] and for the space $L_{p}[-1,1 ;-1,1]$ of the integrable multivariable functions on $[-1,1] \times[-1,1]$ in [12]. Also Gadjiev and Orhan [8] have given a Korovkin type approximation theorem, via statistical convergence, on $L_{p^{-}}$ spaces. Some further results concerning the statistical approximation in the space of locally integrable functions may be found in [5] and [4]. The aim of this paper is to study statistical Korovkin type results for statistically uniformly bounded sequences of positive linear operators which map the space of multivariable integrable functions into itself.

First of all, we recall some basic definitions and notations used in this paper.

Let $A=\left(a_{j n}\right)$ be a nonnegative regular matrix. The $A$-density of $K \subseteq \mathbb{N}$ is given by

$$
\delta_{A}(K):=\lim _{j} \sum_{n \in K} a_{j n} .
$$

The first author was supported by the Scientific and Technological Research Council of Turkey (TUBITAK). 
A sequence $x=\left(x_{n}\right)$ is called $A$-statistically convergent to a number $L$ if for every $\varepsilon>0$,

$$
\delta_{A}\left(\left\{n \in \mathbb{N}:\left|x_{n}-L\right| \geq \varepsilon\right\}\right)=0 .
$$

It is not difficult to see that 1.1 is equivalent to

$$
\lim _{j \rightarrow \infty} \sum_{n:\left|x_{n}-L\right| \geq \varepsilon} a_{j n}=0, \text { for every } \varepsilon>0 .
$$

This limit expression is denoted by $s t_{A}-\lim _{n} x_{n}=L$. The case in which $A=C_{1}$, the Cesàro matrix, reduces to statistical convergence [7].

Let $A=\left(a_{j n}\right)$ be a nonnegative regular matrix. Then the sequence $x=\left(x_{n}\right)$ is said to be strongly $A$-summable to $L$ if

$$
\lim _{j} \sum_{n} a_{j n}\left|x_{n}-L\right|=0 .
$$

By $L_{p}[a, b ; c, d]$ we denote the space of all functions $f$ defined on $[a, b] \times[c, d]$ for which

$$
\int_{c}^{d} \int_{a}^{b}|f(x, y)|^{p} d x d y<\infty, 1 \leq p<\infty .
$$

In this case, the $L_{p}$ norm of a function $f$ in $L_{p}[a, b ; c, d]$, denoted by $\|f\|_{p}$, is given by

$$
\|f\|_{p}:=\left(\int_{c}^{d} \int_{a}^{b}|f(x, y)|^{p} d x d y\right)^{1 / p} .
$$

If $T$ is a positive linear operator from $L_{p}$ into $L_{p}$ then the operator norm $\|T\|_{L_{p} \rightarrow L_{p}}$ is given by

$$
\|T\|_{L_{p} \rightarrow L_{p}}:=\sup _{\|f\|_{p}=1}\|T f\|_{p}
$$

\section{2. $L_{p}$-APPROXIMATION THEOREMS IN STATISTICAL SENSE}

In [12] Zaritskaya has given the following Korovkin type theorem for a uniformly bounded sequence of positive linear operators which map the space $L_{p}[-1,1 ;-1,1]$ into itself.

Theorem 1. Let $\left\{T_{n}\right\}$ be a uniformly bounded sequence of positive linear operators from $L_{p}[-1,1 ;-1,1]$ into itself. Then convergence of the sequence $\left\{T_{n} f\right\}$ to $f$ in $L_{p}$ norm holds for any function $f \in L_{p}[-1,1 ;-1,1]$ if and only if

$$
\lim _{n}\left\|T_{n}\left(f_{i} ; x, y\right)-f_{i}(x, y)\right\|_{p}=0, i=1,2,3,4
$$

where $f_{1}(t, v)=1, f_{2}(t, v)=t, f_{3}(t, v)=v, f_{4}(t, v)=t^{2}+v^{2}$. 
In this section, replacing ordinary "limit" operation by "A-statistical limit" operation, we give an analogues result for Theorem 1.

Theorem 2. Let $A=\left(a_{j n}\right)$ be a non-negative regular summability matrix and let $\left\{T_{n}\right\}$ be an A-statistically uniformly bounded sequence of positive linear operators from $L_{p}[a, b ; c, d]$ into $L_{p}[a, b ; c, d], 1 \leq p<\infty$. Then for any function $f \in L_{p}[a, b ; c, d]$,

$$
s t_{A}-\lim _{n}\left\|T_{n}(f ; x, y)-f(x, y)\right\|_{p}=0
$$

if and only if

$$
s t_{A}-\lim _{n}\left\|T_{n}\left(f_{i} ; x, y\right)-f_{i}(x, y)\right\|_{p}=0, i=1,2,3,4
$$

where $f_{1}(t, v)=1, f_{2}(t, v)=t, f_{3}(t, v)=v, f_{4}(t, v)=t^{2}+v^{2}$.

Proof. It is obvious that 2.2 implies 2.3. To show that 2.3 implies 2.2, let $\left\{T_{n}\right\}$ be an $A$-statistically uniformly bounded sequence of positive linear operators and $f \in L_{p}[a, b ; c, d]$. Then there exist $M>0$ such that $\delta_{A}\left(K_{0}\right)=1$ where $K_{0}:=\left\{n \in \mathbb{N}:\left\|T_{n}\right\|_{L_{p \rightarrow L_{p}}} \leq M\right\}$. Given $\varepsilon>0$, there exist $n_{i}(\varepsilon)$ and $K_{i} \subseteq \mathbb{N}$ of density 1 such that

$$
\left\|T_{n}\left(f_{i} ; x, y\right)-f_{i}(x, y)\right\|_{p}<\varepsilon, i=1,2,3,4
$$

for all $n \in K_{i}$ and $n>n_{i}(\varepsilon)$. Then inequality 2.4 holds for all $n \in K:=\bigcap_{i=0}^{4} K_{i}$ and $n>n_{0}:=\max \left\{n_{i}: i=1,2,3,4\right\}$.

Since $C[a, b ; c, d]$, the set of continuous functions on $[a, b] \times[c, d]$ is dense in $L_{p}[a, b ; c, d]$, for any $\varepsilon>0$ there exists $g \in C[a, b ; c, d]$ such that

$$
\|f(x, y)-g(x, y)\|_{p}<\varepsilon .
$$

Hence for all $n \in K$ and $n>n_{0}$ we have

$$
\begin{aligned}
\left\|T_{n}(f ; x, y)-f(x, y)\right\|_{p} \leq & \left\|T_{n}(f-g ; x, y)\right\|_{p}+\left\|T_{n}(g ; x, y)-g(x, y)\right\|_{p} \\
& +\|f(x, y)-g(x, y)\|_{p} \\
& <\varepsilon(1+M)+\left\|T_{n}(g ; x, y)-g(x, y)\right\|_{p} .
\end{aligned}
$$

By the continuity of $g$ on $[a, b] \times[c, d]$, for any $\varepsilon>0$ there exists $\delta>0$ such that for all $(x, y),(t, v) \in[a, b] \times[c, d]$ satisfying $(t-x)^{2}+(v-y)^{2}<\delta^{2}$ we have

$$
|g(t, v)-g(x, y)|<\varepsilon .
$$

Note that

$$
\begin{aligned}
|g(t, v)-g(x, y)| & \leq|g(t, v)|+|g(x, y)| \\
& <\frac{2 H}{\delta^{2}} \phi(t, v)
\end{aligned}
$$


for all $(x, y),(t, v) \in[a, b] \times[c, d]$ satisfying $(t-x)^{2}+(v-y)^{2} \geq \delta^{2}$ where $\phi(t, v):=(t-x)^{2}+(v-y)^{2}$ and $H=\|g(x, y)\|_{C[a, b ; c, d]}$. Then for any $(x, y)$, $(t, v) \in[a, b] \times[c, d]$ we also have

$$
|g(t, v)-g(x, y)|<\varepsilon+\frac{2 H}{\delta^{2}} \phi(t, v) .
$$

On the other hand one can get

$$
\begin{aligned}
\left\|T_{n}(g ; x, y)-g(x, y)\right\|_{p} \leq & \left\|T_{n}(|g(t, v)-g(x, y)| ; x, y)\right\|_{p} \\
& +H\left\|T_{n}\left(f_{1} ; x, y\right)-f_{1}\right\|_{p} .
\end{aligned}
$$

Using the linearity and positivity of the operators $T_{n}$ and inequality 2.6, we get for any $n \in K$ and $n>n_{0}$ that

$$
\begin{aligned}
\left\|T_{n}(|g(t, v)-g(x, y)| ; x, y)\right\|_{p} \leq & \left\|T_{n}\left(\varepsilon+\frac{2 H}{\delta^{2}} \phi(t, v) ; x, y\right)\right\|_{p} \\
\leq & \varepsilon\left(\left\|T_{n}\left(f_{1} ; x, y\right)-f_{1}\right\|_{p}+1\right) \\
& +\frac{2 H}{\delta^{2}}\left\|T_{n}(\phi(t, v) ; x, y)\right\|_{p} \\
\leq & \varepsilon\left(\left\|T_{n}\left(f_{1} ; x, y\right)-f_{1}\right\|_{p}+1\right) \\
& +\frac{2 H}{\delta^{2}}\left\{\left\|T_{n}\left(f_{4} ; x, y\right)-f_{4}\right\|_{p}\right. \\
& +\left(\alpha^{2}+\beta^{2}\right)\left\|T_{n}\left(f_{1} ; x, y\right)-f_{1}\right\|_{p} \\
& +2 \alpha\left\|T_{n}\left(f_{2} ; x, y\right)-f_{2}\right\|_{p} \\
& \left.+2 \beta\left\|T_{n}\left(f_{3} ; x, y\right)-f_{3}\right\|_{p}\right\}
\end{aligned}
$$

where $\alpha=\max \{|a|,|b|\}$ and $\beta=\max \{|c|,|d|\}$. It follows from 2.5, 2.7 and 2.8, for all $n \in K$ and $n>n_{0}$, that

$$
\begin{aligned}
\left\|T_{n}(f ; x, y)-f(x, y)\right\|_{p} \leq & \varepsilon(2+M) \\
& +\left(H+\varepsilon+\frac{2 H}{\delta^{2}}\left(\alpha^{2}+\beta^{2}\right)\right)\left\|T_{n}\left(f_{1} ; x, y\right)-f_{1}\right\|_{p} \\
& +\frac{4 H \alpha}{\delta^{2}}\left\|T_{n}\left(f_{2} ; x, y\right)-f_{2}\right\|_{p} \\
& +\frac{4 H \beta}{\delta^{2}}\left\|T_{n}\left(f_{3} ; x, y\right)-f_{3}\right\|_{p} \\
& +\frac{2 H}{\delta^{2}}\left\|T_{n}\left(f_{4} ; x, y\right)-f_{4}\right\|_{p} .
\end{aligned}
$$

Using 2.3, inequality 2.9 can be made small enough for all $n \in K$ and $n>n_{0}$. Hence, we have

$$
s t_{A}-\lim _{n}\left\|T_{n}(f ; x, y)-f(x, y)\right\|_{p}=0
$$


which concludes the proof.

Since any bounded $A$-statistically convergent sequence is strongly $A-$ summable [9], the following result holds immediately.

Corollary 1. Let $A=\left(a_{j n}\right)$ be a nonnegative regular summability matrix and let $\left\{T_{n}\right\}$ be a uniformly bounded sequence of positive linear operators from $L_{p}[a, b ; c, d]$ into $L_{p}[a, b ; c, d]$ satisfies

$$
\lim _{n}\left\|T_{n}\left(f_{1} ; x, y\right)-f_{1}(x, y)\right\|_{p}=0
$$

and

$$
s t_{A}-\lim _{n}\left\|T_{n}\left(f_{i} ; x, y\right)-f_{i}(x, y)\right\|_{p}=0, i=2,3,4 .
$$

Then for any function $f \in L_{p}[a, b ; c, d]$

$$
\lim _{j \rightarrow \infty} \sum_{n=1}^{\infty} a_{j n}\left\|T_{n}(f ; x, y)-f(x, y)\right\|_{p}=0 .
$$

Now using the same methods as in the proof of Theorem 2, one can get the following result easily.

Theorem 3. Let $A=\left(a_{j n}\right)$ be a non-negative regular summability matrix and let $\left\{T_{n}\right\}$ be an A-statistically uniformly bounded sequence of positive linear operators from $L_{p}[J]$ into $L_{p}[J], 1 \leq p<\infty$. Then for any function $f \in L_{p}[J]$,

$$
s t_{A}-\lim _{n}\left\|T_{n} f-f\right\|_{p}=0
$$

if and only if

$$
s t_{A}-\lim _{n}\left\|T_{n} f_{i}-f_{i}\right\|_{p}=0, i=1,2, \ldots, m+2
$$

where $f_{1}\left(t_{1}, \ldots, t_{m}\right)=1, \quad f_{i}\left(t_{1}, \ldots, t_{m}\right)=t_{i}, \quad(i=2,3, \ldots, m+1)$, $f_{m+2}\left(t_{1}, \ldots, t_{m}\right)=\sum_{k=1}^{m} t_{k}^{2}$ and $J:=J_{1} \times J_{2} \ldots \times J_{m}, J_{i}=\left[a_{i}, b_{i}\right], i=1,2,3, \ldots, m$.

In this theorem if we choose;

i) $m=1$ and $A=I$, the identity matrix, we get Dzyadyk's result [6].

ii) $m=1$ and $A=C_{1}$, the Cesàro matrix, we get Theorem 7 in [8].

iii) $m=2$ and $A=I$, we get Theorem 1 .

iv) $m=2$, we get Theorem 2 .

Remark 1 . Let $A=\left(a_{j n}\right)$ be a non-negative regular summability matrix for which $\lim _{j} \max _{n} a_{j n}=0$. Then it is well known that $A$-statistical convergence is stronger than ordinary convergence [9]. We can choose a non-negative $A$-statistically null but non-convergent sequence $\left(\mu_{n}\right)$. Let the operators $U_{n}$ on $L_{p}[-1,1 ;-1,1]$ be defined by

$$
U_{n}(f ; x, y)=\left(1+\mu_{n}\right) T_{n}(f ; x, y)
$$


for all $f \in L_{p}[-1,1 ;-1,1]$ where

$T_{n}(f ; x, y)=\frac{1}{1+2^{-n}}\left\{\begin{array}{ll}f(x, y), & 2^{-n} \leq|x| \leq 1 \text { or } 2^{-n} \leq|y| \leq 1 \\ \frac{1}{4} \int_{-1}^{1} \int_{-1}^{1} f(x, y) d x d y, & |x|<2^{-n} \text { and }|y|<2^{-n}\end{array}\right.$.

To show that $\left\{U_{n}\right\}$ satisfies Theorem 2 but it does not satisfy Theorem 1, we will show that $\left\{T_{n}\right\}$ is uniformly bounded sequence satisfying condition (2.1). A simple calculation shows that for all $n \in \mathbb{N},\left\|T_{n} f\right\|_{p}<8^{1 / p}\|f\|_{p}$ and $\left\|T_{n}\right\|_{L_{p} \rightarrow L_{p}}<8^{1 / p}$. Hence $\left\{T_{n}\right\}$ is a uniformly bounded sequence of positive linear operators from $L_{p}[-1,1 ;-1,1]$ into $L_{p}[-1,1 ;-1,1], 1 \leq p<\infty$. Also it is easy to verify that

$$
\begin{gathered}
\left\|T_{n}\left(f_{1} ; x, y\right)-f_{1}(x, y)\right\|_{p}=4^{1 / p}\left(1-\frac{1}{1+2^{-n}}\right), \\
\left\|T_{n}\left(f_{2} ; x, y\right)-f_{2}(x, y)\right\|_{p}=\left\|T_{n}\left(f_{3} ; x, y\right)-f_{3}(x, y)\right\|_{p} \\
=\left\{\frac{4}{p+1}\left[\left(1-\frac{1}{1+2^{-n}}\right)^{p}\left(1-2^{-n}\right)+2^{-n(p+2)}\right]\right\}^{1 / p}
\end{gathered}
$$

and

$$
\left\|T_{n}\left(f_{4} ; x, y\right)-f_{4}(x, y)\right\|_{p}<\left[\left(1-\frac{1}{1+2^{-n}}\right) 2^{p+3}+2^{p-n} 16\right]^{1 / p} .
$$

Hence we necessarily have

$$
\lim _{n}\left\|T_{n}\left(f_{i} ; x, y\right)-f_{i}(x, y)\right\|_{p}=0, i=1,2,3,4 .
$$

\section{REFERENCES}

[1] F. Altomare and M. Campiti, Korovkin-type approximation theory and its applications, ser. de Gruyter Studies in Mathematics. Berlin: Walter de Gruyter, 1994, vol. 17.

[2] H. Berens and R. De Vore, "Quantitative Korovkin theorems for positive linear operators on $L_{p^{-}}$ spaces," Trans. Am. Math. Soc., vol. 245, pp. 349-361, 1978.

[3] H. Berens and R. A. DeVore, "Quantitative Korovkin theorems for $L_{p}$-spaces," in Approx. Theory II. Austin: Proc. int. Symp., 1976, pp. 289-298.

[4] O. Duman and C. Orchan, "Statistical approximation in the space of locally integrable functions," Publ. Math., vol. 63, no. 1-2, pp. 133-144, 2003.

[5] O. Duman and C. Orhan, "Rates of $a$-statistical convergence of operators in the space of locally integrable functions," Appl. Math. Lett., vol. 21, no. 5, pp. 431-435, 2008.

[6] V. K. Dzyadyk, "Approximation of functions by positive linear operators and singular integrals," Mat. Sb., pp. 508-517, 1966.

[7] J. A. Fridy, “On statistical convergence," Analysis, vol. 5, pp. 301-313, 1985.

[8] A. D. Gadjiev and C. Orhan, "Some approximation theorems via statistical convergence," Rocky Mt. J. Math., vol. 32, no. 1, pp. 129-138, 2002.

[9] E. Kolk, "Matrix summability of statistically convergent sequences," Analysis, vol. 13, no. 1-2, pp. 77-83, 1993.

[10] P. P. Korovkin, Linear operators and approximation theory. Delhi: Hindustan Publ., 1960. 
[11] J. J. Swetits and B. Wood, "On the degree of $L_{p}$ approximation with positive linear operators," $J$. Approximation Theory, vol. 87, no. 2, pp. 239-241, 1996.

[12] Z. V. Zaritskaya, "Approximation of functions of two variables by positive linear operators in the L $p$ metric," Ukr. Math. J., vol. 25(1973), pp. 298-302, 1974.

\section{Authors' addresses}

\section{İlknur Sakaoğlu}

Ankara University, Department of Mathematics, Ankara, Turkey

E-mail address: i.sakaogluegmail.com

\section{Mehmet Ünver}

Ankara University, Department of Mathematics, Ankara, Turkey

E-mail address: munverescience.ankara.edu.tr 